\title{
Former à la communication de la science : enjeux et pratiques en europe
}

Olivier Laügt

\section{(2) OpenEdition}

1 Journals

Édition électronique

URL : http://journals.openedition.org/communicationorganisation/2091

DOI : 10.4000/communicationorganisation.2091

ISSN : $1775-3546$

Éditeur

Presses universitaires de Bordeaux

Édition imprimée

Date de publication : 1 novembre 1998

ISSN : 1168-5549

Référence électronique

Olivier Laügt, « Former à la communication de la science : enjeux et pratiques en europe »,

Communication et organisation [En ligne], 14 | 1998, mis en ligne le 26 mars 2012, consulté le 19 avril

2019. URL : http://journals.openedition.org/communicationorganisation/2091 ; DOI : 10.4000/

communicationorganisation.2091

Ce document a été généré automatiquement le 19 avril 2019

(c) Presses universitaires de Bordeaux 


\title{
Former à la communication de la science : enjeux et pratiques en europe
}

\author{
Olivier Laügt
}

1 Dans notre société, la science joue un rôle essentiel en tant qu'argument d'autorité. Quand, dans un processus technocratique, échoit à un expert le pouvoir de trancher au nom de la vérité objective, il va en fait orienter une décision selon aussi des critères politiques, souvent passés sous silence au profit de raisons scientifiques. Il est alors investi d'un pouvoir exorbitant, car il exerce ainsi un pouvoir politique qui ne peut être soumis au contrôle social.

2 L'idéal démocratique nécessite alors, pour échapper à cette domination, que les citoyens soient en mesure de mettre à jour, de peser et de relativiser toutes ces raisons. Or les raisons scientifiques ne peuvent être véritablement maîtrisées que par les spécialistes. Les processus d'information des publics sur les sujets scientifiques, de communication de la science, sont donc à ce titre un enjeu essentiel. Nous allons ici préciser cet enjeu, et examiner comment sont formés les professionnels qui assument ces tâches.

\section{La connaissance scientifique}

Un sens commun sur la science veut qu'il existe des lois de la nature. Ce point de vue, rassurant, suppose alors l'existence d'une vérité cachée qu'il faut découvrir, transformant l'activité de recherche en un gigantesque jeu de pistes.

Les travaux en philosophie et en sociologie des sciences mettent cette vision à mal. Nos théories, ou nos modèles, n'apparaissent alors plus que comme des représentations, des outils plus ou moins adéquats pour répondre à un questionnement, pour résoudre des difficultés. Les sciences vont alors faire succéder, et parfois aussi coexister, diverses interprétations du monde. Cela n'est pas incohérence, mais prise en compte de projets différents. 
5 La question se pose alors de l'aune à laquelle juger la validité d'une théorie. Et bien sûr, différentes approches se trouvent en concurrence, se complétant mutuellement au-delà de leurs oppositions. Un courant classique met l'accent sur la rationalité de la démarche scientifique, et suppose l'existence d'une démarcation entre systèmes d'énoncés scientifiques, et systèmes non scientifiques. Le critère est alors celui de la falsifiabilité (Popper 1959). Le progrès de la science consiste alors à abandonner un modèle falsifié, à l'issue d'une expérience cruciale, pour le remplacer par une nouvelle théorie, qui n'a d'intérêt que dans la mesure où elle autorise des prédictions nouvelles, qui vont être confirmées.

6 Les travaux de la nouvelle sociologie des sciences vont, eux, prendre en compte le caractère historique de l'évolution des idées, et à la limite ne plus guère distinguer la science d'autres réalisations culturelles. La communauté scientifique est alors un groupe social qui, en acceptant un énoncé, lui confère le statut de fait scientifique. Un paradigme (Kuhn 1962) définit alors une norme de travail, coordonne et étaye une tradition de science. Lorsque des anomalies graves et répétées provoquent une période de crise, une révolution scientifique peut avoir lieu, et un paradigme rival vient supplanter l'ancien.

7 Une vision provocatrice et anarchiste de la connaissance a même été développée par Paul Feyerabend qui soutient que compte tenu de la complexité de l'histoire des sciences, il n'est pas possible de dégager de règle à la validité universelle, et que face aux limites des méthodologies, seul survit le «tout est bon» (1979, p. 333). Cela permet enfin à Feyerabend de dégager une perspective humaniste, et de récuser le caractère obligatoire de l'enseignement des sciences à l'école, en revendiquant une séparation de l'État et de la Science, au même titre qu'entre l'Église et l'État.

8 Il ressort alors de ces différents points de vue, au-delà de leurs divergences, qu'il n'existe pas dans le domaine scientifique de certitude absolue, que toute connaissance n'est que provisoire, erreur en sursis, plus ou moins appropriée à certains contextes. Mais malgré cela, le discours scientifique est naturellement investi d'un caractère d'autorité.

9 L'autorité peut se définir comme une supériorité de mérite ou de séduction qui impose l'obéissance, le respect ou la confiance (dictionnaire Robert). Il est aisé de constater l'utilisation de l'autorité de la science comme argument, éventuellement de façon abusive. La publicité fourmille d'exemples de ce type, prétendant, vues de laboratoire et tubes à essai à l'appui, qu'il a été démontré scientifiquement que telle lessive lave plus blanc. Il convient alors de se demander quel est le mérite de la science, quelle est la séduction qu'elle déploie pour s'affirmer comme autorité. Pierre Bourdieu (1982) invite à chercher les racines de l'autorité d'un discours dans les conditions sociales de sa production.

10 La connaissance scientifique se construit par la controverse. Un fait expérimental n'a d'intérêt scientifique que lorsqu'il est connecté à une théorie, ouvrant alors la porte à une interprétation. Le chercheur, travaillant dans un cadre théorique, imagine et construit un dispositif expérimental. Il propose alors une interprétation de ses observations, en utilisant une grille de lecture qu'il a préparée conformément à son a priori théorique. Cette grille isole certains facteurs, néglige les autres. Mais le fait expérimental et son interprétation par un auteur ne pourront fournir une connaissance certifiée que lorsqu'ils auront été soumis à la critique. Cette critique a pour objectif d'assurer qu'il n'y a pas d'autre interprétation possible. Elle se fait en passant le dispositif expérimental au crible, pour s'assurer de l'absence de tout biais. 
11 La controverse doit alors soulever les objections que l'auteur n'a pas devancées. Comme il s'agit donc de sonder la cohérence de l'ensemble constitué par la théorie, l'appareillage de laboratoire et la grille d'analyse, une connaissance profonde de ces outils est requise. Et c'est parce qu'elle met ainsi à l'épreuve les processus expérimentaux, que la discussion n'est pas seulement affaire de logique. Elle exige de ses participants une compétence dans le domaine, ce qui la réserve au cercle restreint des pairs. Ceux-ci sont directement concernés par son issue. En effet, la nouvelle proposition, si elle est acceptée, va étoffer le bagage théorique commun, en ouvrant de nouvelles pistes de recherche ou en en refermant d'autres. L'ensemble de la communauté a donc intérêt à ce que la controverse soit de qualité, ce qui rendra son résultat fiable (Stengers 1997).

La connaissance certifiée qui se construit de cette manière revêt alors un caractère d'objectivité. Il n'est pas fondé sur une quelconque idée d'impartialité surhumaine, propre aux hommes de science, qui les placerait à l'écart du commun des mortels. Il ne repose pas plus sur le lieu commun de leur interchangeabilité, qui conduit à dire que si Newton n'avait pas découvert la gravitation universelle, un autre l'aurait fait dans les mêmes termes, alors que nul autre que Picasso n'aurait pu peindre Guernica. Ce caractère d'objectivité repose sur la publicité et le processus communicationnel qui président à la construction de cette connaissance.

13 Le discours scientifique qui en résulte offre ainsi une garantie de rationalité et de cohérence. Il se situe ainsi nettement par rapport à la ligne qui partage raison de folie, et appartient clairement de ce fait, selon Michel Foucault (1971), à la catégorie des discours autorisés. Et plus, la volonté de savoir, la quête de vérité qui le sous-tend font qu'il domine et contraint, toujours en suivant Foucault, tous les autres discours. En cela donc la science impose obéissance, si ce n'est respect ou totale confiance, et par conséquent fait autorité.

Un décalage apparait donc entre la nature de la connaissance scientifique et ce caractère d'autorité du discours scientifique, qui nourrit le sens commun sur la science évoqué plus haut. On peut alors s'interroger sur la relation de notre société à la science.

\section{La relation de la société à la science}

En Occident, la représentation du monde a été bouleversée par l'abandon des conceptions aristotéliciennes. Le Moyen Age, imprégné de religion, ne s'autorisait pas à expliquer l'univers, mais tentait seulement de le décrire. Depuis la Renaissance, à la suite de l'audace de Galilée, nous osons des tentatives d'explications. Le Cosmos n'est plus sagement ordonné dans un espace clos, gouverné par les dieux et caisse de résonance des mythes, mais se représente comme un ciel vide en expansion infinie, et aux lois mathématiques.

La science se trouve depuis à la base de la stratégie de croissance et de développement de toutes les nations. Elle est partout présente dans notre vie quotidienne. Pourquoi cette emprise? Parce que le sens commun va à son propos évoquer un savoir objectif, universel, prouvé, et parce qu'une assimilation est faite par le public entre science, comprise comme accumulation de vérités, et progrès social, communément pris comme toujours plus de biens et de justice.

17 Au-delà de ses premiers sens de mouvement en avant et d'évolution (dictionnaire Robert), le terme de progrès porte une connotation positive, renvoyant à une idée 
d'amélioration et d'évolution vers un terme idéal. Il s'agit là d'une catégorie spécifique au monde occidental, qui nécessite d'envisager le futur en référence à un objectif. Cette notion est susceptible de s'altérer et de virer à une idéologie du progrès lorsqu'elle laisse la place à l'idée vague d'un progrès total, vide de contenu et impossible à vraiment définir (Droit 1990).

Or, depuis la Renaissance, l'idée de progrès cumulatif a été appliquée à l'avancement des sciences. Ce courant, à la suite de Condorcet, de Comte ou de Spencer, a conduit à associer progression des connaissances et progression sociale de l'humanité, et à croire au Progrès (Lecourt 1997). Le scientisme a cru que le développement scientifique amènerait l'homme à être plus éclairé, plus moral, à maîtriser rationnellement les problèmes sociaux.

À côté de cela, la progression des techniques et les réussites de La science ont accentué La tendance à la confusion, confusion entre avancement technique et progrès social, confusion entre science et technique. Pour le public, les figures du savant ou du chercheur sont proches de celles de l'ingénieur ou du médecin. L'intrication entre l'évolution des techniques d'observation ou de manipulation du monde d'une part, et l'évolution des connaissances d'autre part, favorise cette assimilation. Lors de la révolution industrielle par exemple, la domestication de la vapeur, qui facilite les communications et fait progresser l'industrie, suscite l'étude de la thermodynamique et en bénéficie en retour. D'un autre côté, le perfectionnement des techniques supporte l'amélioration de la condition humaine. Il suffit de penser aux progrès de la médecine, à Pasteur «bienfaiteur de l'humanité » : l'application des connaissances participe au bienêtre général.

De cette confusion est né au dix-neuvième siècle l'amalgame entre évolution de la connaissance scientifique et accession à plus de bonheur, de santé ou de bien-être. Mais le vingtième siècle dément cette illusion. Il apparaît que loin de résoudre tous les problèmes, sciences et technologies en créent de nouveaux. On peut situer la source de la déshumanisation de la connaissance dans l'engagement largement volontaire des scientifiques dans le projet Manhattan. Ce programme, proposé par certains d'entre eux, comme Fermi et Szilard, pour utiliser leurs connaissances à la mise au point de la bombe atomique avant que l'Allemagne hitlérienne n'y parvienne, aboutira aux milliers de morts et de mutilés d'Hiroshima et de Nagasaki. La menace nucléaire, ressentie dès lors par l'humanité entière, est associée à la science. Depuis, la science, inséparable de ses applications qui seules sont connues du grand public, perpétue une image contrastée de bien et de mal. Ses applications visibles mêlent des progrès spectaculaires et des catastrophes technologiques. Les pollutions des eaux, par marées noires ou rejets de produits toxiques dans des rivières, sont monnaie courante. Les accidents de l'industrie chimique à Seveso (1976) ou à Bhopal (2 500 morts en 1984) restent dans les mémoires. Les explosions nucléaires (à Three Mile Island en 1979, à Tchernobyl en 1986) marquent l'opinion. Dans le domaine biologique et médical, la connaissance scientifique allie des avancées fantastiques à des dérives. Elle allonge la vie, en greffant et en guérissant, au moins pour ceux qui en ont les moyens. Mais elle engendre aussi des dérapages (affaire du sang contaminé), ou en laisse craindre (choix éthiques liés aux grossesses démarrées in vitro, organismes génétiquement modifiés, possibilités de clonage).

21 L'attitude du public vis à vis de la science et de la technique est donc maintenant plus ambiguë. Les analyses de l'Eurobaromètre 46.1, enquête financée en 1996 par la Commission européenne (DG12), montrent qu'en Europe espoirs et craintes sont mêlés vis à vis des biotechnologies, même si en fin de compte le public les soutient, en pensant 
qu'elles auront des retombées positives. Plus particulièrement pour la France, les enquêtes du Centre d'étude de la vie politique française (Cevipof), menées de 1972 à 1994, mettent en évidence la croissance, puis la stabilisation, des craintes liées à la préservation de l'environnement (Boy 1995). À côté de cela, sciences et techniques sont massivement perçues comme améliorant les conditions de travail. Mais parallèlement, le progrès technique fait globalement craindre pour l'emploi, même si ce sentiment est variable d'une couche culturelle à une autre. Enfin, il est quasi unanimement reconnu que les changements scientifiques et techniques rendent la vie plus facile. Cela est associé à une nette frustration liée à l'incompréhension des principes de fonctionnement des objets de la vie quotidienne, du lecteur de disques laser à la boîte de vitesses d'une automobile. En définitive, et même si un glissement se fait sentir vers une attitude plus pessimiste, il apparait que le public estime que la science ou le progrès technique lui apportent plus de bien que de mal. Et la représentation des chercheurs comme des gens dévoués qui travaillent pour le bien de l'humanité, avec les figures emblématiques de Pasteur ou de Pierre et Marie Curie, est toujours forte.

L'assimilation entre science et progrès, née des Lumières, et qui a connu son apogée dans le scientisme, est donc encore active dans notre société. Elle confère par conséquent un enjeu fort à la présentation, à la communication de la science

\section{Les enjeux de la communication de la science}

Les deux principaux modes de diffusion de la science, hormis la communication interne à la communauté scientifique, sont l'enseignement et la vulgarisation. Mais cette catégorisation reste sommaire. D'une part elle méconnaît les transitions fines qui peuvent exister entre ces deux pôles, mais elle fait également fi d'autres modes de propagation, comme les relations à la connaissance qui sont induites par l'expertise, lorsqu'un discours d'expert est sollicité pour objectiver une décision.

Les objectifs attribués de prime abord à la communication de la science sont de faire circuler les savoirs, de partager la connaissance. Il s'agit là pour la société qui finance la recherche scientifique d'obtenir des chercheurs un retour sur investissement par la production d'innovations, par une contribution à la santé publique. Il est alors question de transferts de technologies pour que les entreprises restent compétitives, et l'enjeu est économique. Il est par exemple clairement affiché dans les documents de présentation du groupe de recherche Media and Science de l'Université de Sheffield, qui annoncent qu'il s'agit là d'un objectif que le gouvernement considère comme essentiel pour le maintien de la position britannique en tant que leader de la communauté scientifique. Mais au-delà de la valorisation de la recherche au sens strict du terme, on peut voir dans cet objectif de partage de connaissances, la possibilité de mobilisation et d'organisation pour les publics de savoirs fragmentés (Castagna 1997). L'enjeu est dans ce cas démocratique, car il s'agit alors de donner aux citoyens la possibilité de participer à l'espace de négociation qui s'ouvre entre les experts, porte-parole de la rationalité scientifique, et les instances de décision politique (Roqueplo 1992). C'est dans cette optique que le groupe Media and Science travaille plus particulièrement sur la communication autour des biotechnologies, à cause de leurs conséquences du point de vue alimentaire. Se pose en effet la question de ce que l'on donne à voir et à comprendre au public des choses de la science, par la vulgarisation, mais aussi par la médiatisation des controverses, par les querelles 
d'experts, par les annonces de décision. C'est l'axe actuel de recherche de l'équipe JERICOST de l'IUT de Tours (Castagna 1998).

La diffusion de la connaissance scientifique peut aussi correspondre à une intention de permettre à chacun de s'adapter et de maitriser son environnement. Il y a là un enjeu écologique, et un rôle d'éducation sociale (Jeanneret 1994). Mais la difficulté réside alors dans la compréhension du processus de communication, qui ne saurait se schématiser dans une métaphore de traduction, comme si le langage de spécialité (technolecte) n'était qu'un jargon qu'il suffirait de décoder. Par ailleurs, en présentant essentiellement les réponses de la science, les médias doivent reformuler le problème scientifique pour le faire correspondre aux interrogations vraisemblables du public (Jurdant 1969). Cette distorsion ne fera donc que conforter sa vision du monde, en la validant par les résultats scientifiques. Le groupe de recherches en journalisme scientifique de la Freie Universität Berlin mène actuellement un programme de travail sur ce thème, en s'intéressant à la réception médiatique et à la prise en charge personnelle des patients affectés d'une maladie chronique (Medienthema Krebsmedizin).

Mais aussi, en oubliant les conditions de création de la connaissance scientifique, la vulgarisation la dénature et ne devient plus qu'une exhibition (Roqueplo 1974). Elle peut alors avoir pour conséquence d'aboutir à une équivoque sur la nature même des connaissances. Celles-ci se construisent en effet en fusionnant pratique théorique et expérimentation, et en progressant par essais et erreurs. Une vérité scientifique n'est donc qu'une erreur en sursis. Le doute, au sens de remise en question, est donc banal pour le scientifique, et pratique normale. Mais en montrant la connaissance, et en ne la démontrant pas, la vulgarisation transforme pour le public incertitude scientifique en incertitude synonyme d'erreur. Et le public qui échapperait à cette Charybde du spectacle de la science trouve alors, en guise de Scylla, un spectacle sur la science, mise en perspective de l'abîme qui le sépare des scientifiques. Il pourra ainsi prendre la mesure péjorative du terme même de vulgarisation, qui a été préféré par la langue française à son concurrent "popularisation », même si Jean Rostand a essayé de faire passer cette pilule en établissant une analogie avec la traduction de la Bible nommée Vulgate (Raichvarg et Jacques 1991). Il n'a plus alors qu'à réaliser qu'il est couramment qualifié de public profane $^{1}$, ce qui le place dans une position d'exclu du temple, en regard du grand prêtre scientifique. La mythification de la science est alors en place, et en augmentant «la distance entre les profanes et les oracles de la vérité sacralisée »(Sylvestre 1993, p. 1517), la vulgarisation accentue l'autorité naturelle de la science.

Face à l'importance des enjeux, et à l'ampleur des difficultés de la communication de la science, se pose alors la question des différentes formations offertes à ceux qui désirent accéder aux professions de journaliste scientifique, ou de responsables de communication d'organisations intervenant dans le secteur scientifique. À qui s'adressent-elles? Comment préparent-elles à ces tâches?

\section{Les formations à la communication de la science et au journalisme scientifique}

Il s'agit alors d'engager, à l'aide d'une grille d'analyse, une observation de ces formations. Cela signifie donc faire des comparaisons, pour relever traits communs et divergences. Mais la difficulté est alors de ne comparer que ce qui est comparable. Une étude à 
l'échelon international se heurte immédiatement à des obstacles relevant des différences culturelles. Les regards sur la communication changent d'un pays à l'autre. L'organisation et la relation aux études universitaires sont variables. Les façons d'envisager les professions, et la professionnalisation des formations sont également relatives. Une étude faite sur l'appréciation portée sur leur expérience de séjour à l'étranger par des étudiants en communication et cinéma (Martineau 1995) met bien ces disparités en scène, en soulignant le caractère spécifique de chaque université. Un choix est alors fait de limiter la présente étude à l'Europe, en ayant de plus conscience de la non-coïncidence des cultures et des territoires nationaux, et du problème des articulations que cela pose (Bougnoux 1995).

Pour constituer un corpus, une documentation a été rassemblée, constituée de brochures papier de présentation des formations, de pages web des universités et écoles d'Europe, et de renseignements sollicités directement auprès d'enseignants. L'observation ne pourra par conséquent porter que sur ce qui est dit, affiché, annoncé, ce qui en limite la portée. Il ne nous a par ailleurs été personnellement possible que de travailler en langue allemande, anglaise ou française. La documentation disponible est fréquemment bilingue, langue vernaculaire et anglais, mais cela pose aussi le problème de la fidélité de la traduction.

30 En extrayant de cette documentation ce qui concerne les formations au journalisme scientifique ou à la communication de la science, il apparaît immédiatement qu'il existe des cursus spécifiques. Ceux-ci sont d'une part nettement distinguables des éventuelles sensibilisations à la science qui peuvent être offertes dans des formations plus générales, et de plus ne sont pas systématiquement proposés à côté de celles-ci. Par exemple, la City University de Londres ne propose qu'un cours optionnel en science, au choix face à la santé, la finance ou le sport. D'un poids de 100 sur 800 dans les PGDip de journalisme international, télévisuel, presse quotidienne ou magazine entre lesquels il est commun, il est consacré au rôle du correspondant spécialisé, à la connaissance du domaine, et à la constitution de contacts. L'Université de Hambourg également, qui ne propose des formations au journalisme qu'en formation complémentaire à côté d'une formation principale (Nebenfach), n'offre simplement qu'un cours de journalisme scientifique, au niveau d'un premier cycle. En ouvrant une formation spécifique et en réorganisant ses cursus, l'Université de Dublin supprime ce domaine de ses cours menant au Bachelor Degree (4 ans d'études).

31 L'observation ne va donc porter que sur les formations spécialisées. Compte tenu des difficultés évoquées plus haut, la grille d'analyse ne peut être que relativement grossière. Elle va d'abord s'intéresser au public concerné : est-ce une formation de base ou une formation complémentaire, et à quel niveau d'études se situe-t-elle? Elle va ensuite concerner les théories, méthodes et techniques en communication et journalisme : quelle importance leur est donnée, quel est l'accent pratique, y a-t-il un stage qui accompagne la formation? Enfin, on examinera la façon dont sont abordées les questions spécifiques à la communication de la science: propose-t-on une formation ou un complément de formation scientifique ou technique, une réflexion sur la connaissance, ou sur les spécificités de la communication de la science?

Il s'avère alors que ces formations sont sélectives, et ne concernent que des étudiants déjà diplômés dans un cursus scientifique, même si souvent l'accès est possible en justifiant d'une expérience professionnelle. De ce fait, elles constituent des études débouchant sur un Master, ou troisième cycle français. À la FU Berlin, ces formations sont proposées en 
Grundstudium et en Hauptstudium, soit réparties sur les quatre premières années d'études universitaires, mais en complément d'une autre valence. L'Université catholique de Louvain fait alors figure d'exception, qui propose la «médiation des savoirs » comme domaine d'application de sa licence en communication (quatre ans d'études), à côté de la "médiation institutionnelle». Mais cette université propose également des formations complémentaires en communication de la science pour des Licenciés d'autres départements.

L'intégration ou non d'un stage professionnel dans le cursus semble correspondre étroitement à la distinction entre formations au journalisme scientifique et formations à la communication de la science. Les formations au journalisme, qui en exigent toutes d'une durée variant entre deux et quatre mois, mettent rapidement l'accent sur l'aspect technique: ateliers d'écriture, initiation radio et télévision, réalisation d'émissions ou confection d'un périodique. Les formations à la communication de la science restent en revanche plus facilement centrées sur les aspects théoriques.

Mais la divergence principale qui semble pouvoir être distinguée entre ces formations ne recouvre pas, elle, une opposition journalisme vs communication.

Les unes privilégient théories, méthodes et techniques d'information et de communication, et les appliquent à un domaine, la science, qui reste peu ou prou considéré comme une boîte noire. Certains cours ou séminaires portent alors sur les causes de l'échec des médias sur des sujets scientifiques, ou sur les stratégies de diffusion de la science. Par exemple, les seuls cours ayant trait à la science dans le DESS Communication et information scientifique, technique et médicale de l'Université Denis Diderot - Paris 7 concernent les enjeux de la recherche, du développement et de l'innovation. Bien sûr, les aspects philosophiques et sociologiques de la science peuvent être traités lors de l'étude des fonctions et stratégies de la communication d'entreprise, lors de la formation à la communication de crise ou lors d'une réalisation journalistique. Néanmoins, ils ne font pas l'objet d'une réflexion spécifique, et ne peuvent donc être abordés qu'en incidente. Autre exemple à la FU Berlin, qui propose explicitement lors de séminaires de réfléchir sur ce qu'est le bon journalisme scientifique (was guter Wissenschafts-journalimus ist) du point de vue des techniques de présentation, ou d'écouter de bonnes et de mauvaises (gute und schlechte) émissions de radio pour élaborer ensuite des critères et se pencher sur les rôles respectifs de la musique, du son et de la parole. Il s'agit donc uniquement de fournir à des scientifiques la maîtrise d'un certain nombre d'outils.

Inversement, d'autres formations privilégient une réflexion sur la science, et sur la représentation qui en est proposée dans les médias. Place est alors faite à des cours sur l'histoire des sciences et des techniques, sur les théories scientifiques contemporaines, sur les rapports entre sciences, technologies et société, sur l'impact social des découvertes scientifiques, ou encore sur les grands courants de la communication de la science. Ces enseignements sont assurés de concert par des scientifiques, des philosophes et des spécialistes de communication.

Réserver cette place pose alors un problème pratique de lourdeur de la formation. Il est résolu par l'Universitat Pompeu Fabra de Barcelone, dans son Màster en Comunicació Cientifica, en supprimant tout cours technique de communication. L'enseignement est alors composé de deux modules, l'un concernant la communication de la science, l'autre relevant d'une spécialité scientifique. À l'opposé, l'ESJ de Lille, qui organise en liaison avec l'Université Lille 1 la formation «Journaliste et scientifique », ne sacrifie rien et 
propose une formation très lourde de plus de 900 heures, comportant des enseignements généraux, spécialisés, techniques et professionnels assurés par d'ESJ, à côté d'un module « culture et actualité scientifique et technique » extrait du DESS Diffusion des Connaissances Scientifiques et Technologiques. À cela s'ajoute un stage de deux mois en presse écrite. Mais par exemple de façon intermédiaire, la Dublin City University et la Queen's University Belfast, pour le MSc in Science Communication qu'elles organisent conjointement, allègent l'aspect technique, sans y renoncer totalement.

Quoi qu'il en soit dans tous ces cas, la réflexion sur la science et sur la relation science société est essentielle, et tente de fonctionner de manière fusionnelle avec la maitrise d'outils. En effet, même à Barcelone, les cours sont organisés en liaison étroite avec le groupe de recherche Observatorio de la Comunicació Cientifica, dont un des thèmes actuels de travail concerne les stratégies et les nouveaux outils pour la diffusion de la science et de la technologie. Il ne s'agit donc pas de formations à la communication pour des scientifiques, mais plus véritablement de formations à la communication de la science, qui par la force des choses concernent en priorité des scientifiques.

Face à une évaluation des choix scientifiques et technologiques qui impliquent directement les citoyens, l'idéal démocratique exige un débat. Sur un sujet sensible de cet ordre, comme les OGM par exemple, réduire la difficulté à un problème de médiation, et travailler sur la présentation de la question scientifique, peut éviter d'amener le débat sur la question elle-même. Or pour un véritable fonctionnement démocratique, toutes les raisons doivent pouvoir être exposées. Etre capable de traiter des limites, des approximations de la connaissance, des glissements logiques est alors un atout. On peut par conséquent estimer, d'une façon peut-être naïvement volontariste, qu'un responsable de communication ou un journaliste scientifique a un rôle à jouer pour participer à rendre plus aptes les publics à ce débat. Il sera sans doute mieux armé pour cela si sa formation a intégré ces aspects, pour l'aider à faire abstraction de la représentation positiviste, qui est encore dominante comme nous l'avons vu.

L'enjeu est donc, face à l'échec avéré de la vulgarisation traditionnelle, un renouvellement de la communication de la science, qui permettrait non pas sans doute de prendre définitivement ses distances avec les simplifications abusives, cela serait utopique, mais d'avoir au moins conscience de ces simplifications.

Toulouse Gérard, Regards sur l'éthique des sciences, Paris, Hachette, 1998

\section{BIBLIOGRAPHIE}

BOUGNOUX Daniel, « Expression identitaire et communication moderne », in SAEZ J-R (dir.), Identités, cultures et territoires, Paris, Desclée de Brouwer, 1995.

BOURDIEU Pierre, Ce que parler veut dire, Paris, Fayard, 1982.

BOY Daniel, « Les Français, la science et la technologie », in Freeman C. et MENDRAS H. (dir.), Le paradigme informatique, Paris, Descartes et Cie, 1995, pp. 123 à 145. 
CASTAGNA Bernard, « Mobilisation, organisation et diffusion des savoirs scientifiques et techniques ; les enjeux d'une problématique de recherche ", in Méthodes et conduites du débat public : à propos des conférences de consensus et des ateliers de scénarios, les cahiers de jericost, $\mathrm{n}^{\circ} 1$, presses de l'Université de Tours, 1997, pp. 9 à 14.

CASTAGNA Bernard, « jerico-st : mieux comprendre l'évolution des relations entre sciences, techniques et sociétés ", in Management par projet et logiques communicationnelles, Communication et organisation $n^{\circ} 13$, Talence, 1998.

CLAESSENS Michel, La technique contre la démocratie, Paris, Seuil, 1998.

DROIT Roger-Pol (dir), Science et philosophie, pour quoi faire? Forum le Monde le Mans, Paris, Le Monde Éditions, 1990. Eurobaromètre 46.1, Les Européens et la biotechnologie moderne, Bruxelles Luxembourg Office des publications officielles des Communautés européennes, 1997.

FEYERABEND Paul, Contre la méthode, esquisse d'une théorie anarchiste de la connaissance, Paris, Seuil, 1979, traduction Jurdant B. et Schlumberger A., 1 ère édition New Left Books, Londres, 1975.

FOUCAULT Michel, L'ordre du discours, Paris, Gallimard, 1971.

FOUREZ Gérard, La construction des sciences, Bruxelles, De Boeck, 3e édition 1996,1 ${ }^{\text {ère }}$ édition 1988.

JEANNERET Yves, Écrire la science, Paris, PUF, 1994.

JURDANT Baudoin, «Vulgarisation scientifique et idéologie », Communications, n 14, Paris, 1969, pp. 150 à 161 .

KUHN Thomas S., La structure des révolutions scientifiques, Paris, Flammarion, 1983, traduction

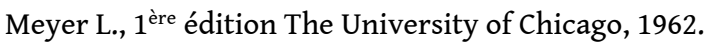

LECOURT Dominique, L'avenir du progrès, Paris, Textuel, 1997.

MARTINEAU Monique (dir.), Regards étudiants sur les échanges : communication et cinéma en Europe, CinémAction $n^{\circ}$ 77, Paris, INRP - Corlet - Télérama - SFSIC, 1995.

POPPER Karl, La logique de la découverte scientifique, Paris, Payot, 1973, traduction Thyssen-Rutten N. et Devaux P., $1^{\text {ère }}$ édition 1959.

RAICHVARG Daniel et Jacques Jean, Savants et ignorants, une histoire de la vulgarisation des sciences, Paris, Seuil, 1991.

ROQUEPLO Philippe, Le partage du savoir, Paris, Seuil, 1974.

ROQUEPLO Philippe, «L'expertise scientifique, consensus ou conflit? » in Theys J. et KALAORA B. (dir.), La terre outragée (les experts sont formels!), Autrement (sciences en société $\left.\mathrm{n}^{\circ}{ }^{\circ} \mathrm{l}\right)$ 1992, Paris, pp. 157 à 169.

STENGERS Isabelle, Sciences et pouvoirs, la démocratie face à la technoscience, Paris, La Découverte, 1997.

SYLVESTRE Jean-Pierre, « Vérité et vulgarisation », in SFEZ L. (dir.), Dictionnaire critique de la communication, Paris, PUF, vol. 2, pp. 1514 à 1518.

\section{NOTES}

1. En anglais, il est question de Lay people, en Allemand de Laie, expressions qui marquent moins une exclusion qu'une situation de droit 


\section{RÉSUMÉS}

Alors que la science est doute et controverse, le discours scientifique est revêtu d'un caractère d'autorité. De plus l'assimilation commune entre science et progrès est encore active dans notre société. Or, à côté des difficultés et de l'échec de la vulgarisation traditionnelle, les enjeux de la communication de la science sont forts. En effet, ils conditionnent la possibilité et la qualité du débat démocratique, dès qu'il est question d'évaluer des choix scientifiques ou technologiques qui concernent les citoyens. Une observation des formations à la communication de la science va permettre alors d'apprécier comment les médiateurs sont préparés à accomplir leur tâche.

While science is doubt and controversy, scientific discourse assume a feature of authority. More, usual assimilation of sciences to progress is still in order in our society. Compared with difficulties and failure of usual scientific popularization, challenge of science communication is important. Indeed, it's conditioning possibility and quality of democratic debate, as soon we're talking about evaluation of scientific or technological choices concerning citizens. A survey of science communication courses will allow to estimate how mediators are trained to do their work.

\section{INDEX}

Mots-clés : communication de la science, débat démocratique, science, vulgarisation

\section{AUTEUR}

\section{OLIVIER LAÜGT}

Olivier Laügt est agrégé de mathématiques, et possède un doctorat en sciences de l'information et de la communication. Enseignant à l'ISIC, ses cours concernent l'application des statistiques aux sciences de l'information et de la communication (traitement d'enquêtes, analyse de discours), et la relation science-société. Son activité de recherche porte principalement sur la rhétorique des discours d'experts et sur son action sur l'image de la science. 